1. Yoshizawa T, Shinmi O, Giaid A, et al. Endothelin : a novel peptide in the posterior pituitary system. Science 1990 ; 247 : 462-4. 2. Stojilkovic SS, Merelli F, Iida T, Krsmanovic LZ, Catt KJ. Endothelin stimulation of cytosolic calcium and gonadotropin secretion in anterior pituitary cells. Science $1990 ; 248$ : 1663-6.

3. Schichiri M, Hirata Y, Kanno K, Ohta K, Emori T, Marumo F. Effect of endothelin-1 on release of arginine-vasopressin from perifused rat hypothalamus. Biochem Biophys Res Commun 1989 ; 163 : 1332-7.

4. Serradeil-Le Gal C, Jouneaux C, SanchezBueno A, et al. Endothelin action in rat liver: Receptors, Free $\mathrm{Ca}^{2}+$ oscillations and activation of glycogenolysis. $J$ Clin Invest 1991; in press.

5. Takuwa $\mathrm{N}$, Takuwa $\mathrm{Y}$, Yanagisawa $\mathrm{M}$, Yamashita K, Masaki T. A novel vasoactive peptide endothelin stimulates mitogenesis through inositol lipid turnover in Swiss 3T3 fibroblasts. J Biol Chem 1989 ; 264 : 7856-61. 6. Simonson MS, Wann S, Mené $\mathrm{P}$, et al. Endothelin stimulates phospholipase C, $\mathrm{Na}+/ \mathrm{H}^{+}$exchange, $c$-fos expression, and mitogenesis in rat mesangial cells. J Clin Invest 1989: $83: 708-12$.

7. Yanagisawa M, Masaki T. Molecular biology and biochemistry of the endothelins. Trends Pharmacol Sci 1989 ; 10 : 374-8.

\section{口曰 BRÈVE $\square$}

La prise en quantité excessive de vitamine A pendant la grossesse pourrait être à l'origine de rares anomalies chez le nouveau-né. Ce risque rapporté dans la revue Toxicology [1] est extrêmement faible mais a conduit cependant les autorités anglaises concernées à charger d'une enquête sur ce sujet le Committee on Toxicily. A la suite des conclusions de cet organisme, le département anglais de la santé invite les femmes enceintes à ne pas consommer de façon excessive de la vitamine $\mathrm{A}$ et en particulier à éviter la consommation régulière de foie. Dans cent grammes de cet abat on trouverait en effet de 4 à 12 fois la dose maximum recommandée pendant la grossesse.

[1. Comparative assessment in the toxicology of vitamin $\mathrm{A}$ and retinoid acid in man. Toxicology $1989 ; 57$ : 117.]

$m / s n^{\circ} 1$, vol. 7 , janvier 91

\title{
Paralysies périodiques hyperkaliémiques et canal sodium musculaire
}

Les paralysies périodiques primitives sont des affections dominantes autosomiques qui s'accompagnent d'une anomalie du potassium sanguin, le plus souvent d'une élévation. La paralysie périodique hyperkaliémique (HYPP) débute en général avant dix ans et se manifeste par des accès de faiblesse musculaire, qui peuvent aboutir à une myopathie vacuolaire. Il en existe plusieurs formes, suivant que s'associe ou non une myotonie. On a pu montrer in vitro qu'une élévation légère de $\mathrm{K}+$ extracellulaire déclenche dans le muscle atteint, et lui seul, un courant $\mathrm{Na}+$ sensible à la tétrodoxine. Comme la sous-unité $\alpha$ du canal sodium suffit à former un canal sodium sensible à la tétrodoxine et dépendant du voltage, une équipe de Boston, MA (USA) (13 auteurs) a fait l'hypothèse que HYPP pourrait être lié génétiquement au locus de cette sous-unité du canal sodium [1]. Ils ont utilisé au départ des clones récemment isolés d'ADNc de rat [2], qui leur ont permis d'obtenir des clones humains correspondant à des domaines intracellulaires. L'un d'eux, long de $600 \mathrm{pb}$, est spécifique du gène de l'isof orme adulte du canal sodium musculaire. Des hybrides somatiques cellulaires ont permis de localiser le gène sur le bras long du chromosome 17 , dont une carte détaillée a été récemment publiée [3]. Les auteurs ont identifié un polymorphisme de restriction à l'enzyme BglII, dont la position sur la carte a été précisée. De plus le gène est étroitement lié à celui de l'hormone de croissance GH1, situé en 17q22-q24 et pour lequel on connaît un site de restriction polymorphe à Hind I. A l'aide de ces deux marqueurs on a exploré une vaste famille atteinte de HYPP avec myotonie ; 42 sujets ont été examinés dont 24 malades. Le lod score était de 7, l'interprétation que la sous-unité $\alpha$ du canal sodium du muscle adulte est le siège de l'anomalie dans cette famille est vraisemblable.

Il reste évidemment beaucoup de chemin à parcourir. D'abord, élucider avec précision le mécanisme moléculaire de l'anomalie et établir ainsi des procédés de diagnostic de HYPP ; étendre l'étude à d'autres familles, du même type myotonique et des autres types, ainsi qu'aux formes hypokaliémiques ; une première indication, dans une famille de cette dernière forme, a été fournie par les auteurs eux-mêmes : ils ont trouvé un recombinant entre la maladie et le canal sodium, suggérant l'existence d'un locus séparé. Enfin, renforçant la notion de liaison étroite entre sousunité $\alpha$ du canal sodium et hormone de croissance, ils rappellent qu'il y a un siècle, G. Clouzot avait rapporté l'observation d'une famille dans laquelle la paralysie périodique coségrégeait avec une taille petite, soulevant l'hypothèse d'une lésion concomitante du gène de l'hormone de croissance.

J.-C.D.

1. Fontaine B, Khurana TS, Hoffman EP, $e t$ al. Hyperkaliemic periodic paralysis and the adult muscle sodium channel $\alpha$-subunit gene. Science $1990 ; 250$ : 1000-2

2. Trimmer JA, Cooperman SS, Tomiko SA. Primary structure and functional expression of a mammalian skeletal muscle sodium channel. Neuron 1989 ; 3 : 33-49.

3. Haines JL, Ozelius LJ, McFarlane H, et al. A genetic linkage map of chromosome 17 . Genomics $1990 ; 8: 1-6$. 